\title{
Novel phases and finite-size scaling in two-species asymmetric diffusive processes
}

\author{
Kwan-tai Leung \\ Institute of Physics, Academia Sinica, \\ Taipei, Taiwan 11529, Republic of China
}

\begin{abstract}
We study a stochastic lattice gas of particles undergoing asymmetric diffusion in two dimensions. Transitions between a low-density uniform phase and highdensity non-uniform phases characterized by localized or extended structure are found. We develop a mean-field theory which relates coarse-grained parameters to microscopic ones. Detailed predictions for finite-size $(L)$ scaling and density profiles agree excellently with simulations. Unusual large- $L$ behavior of the transition point parallel to that of self-organized sandpile models is found.
\end{abstract}

Pacs Numbers: 02.50.Ey, 02.60.Cb, 05.70.Fh, 64.60.Cn 
There is much interest in the studies of lattice gas systems evolving under conservative stochastic dynamical rules伍. When the rules do not satisfy detailed balance condition, intriguing properties atypical of thermal equilibrium arise 2]. Their mappings onto surface growth models and directed polymer in a random medium [3] generates further interests and development. Very recently, such connections have

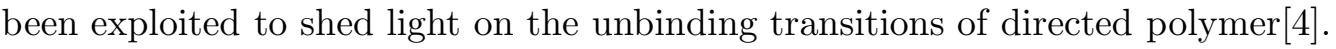

One particularly simple class of lattice gas models consists of particles undergoing biased diffusion with no interaction except hard-core exclusion. Generally known as the asymmetric simple exclusion process (ASEP)[1], it has attracted the interests of both physicists and mathematicians for its novel phase transitions [5] and shock structures [6] under various boundary conditions. Generalizing the ASEP to a model having two species biased along opposite directions in an $L_{x} \times L_{y}$ system, Schmittmann et al.[7] observed a transition between a uniform and a non-uniform phase as the number of particles is varied, with the latter phase characterized by a compact strip of aggregated particles. However, the fundamental question of the existence of the transition in the thermodynamic limit has not been answered. This is not a simple issue to settle, since the limiting process depends crucially on anisotropic finite-size effects induced by making one direction special. The precise form of anisotropies is unknown. Nevertheless, a hint is provided by analogous continuous phase transitions, which typically have ensemble averages depending on $L_{x}^{p} / L_{y}$ with $p \neq 1[8]$. Depending on how $L_{x} \rightarrow \infty$ and $L_{y} \rightarrow \infty$ are taken, undesired singularities may arise from $L_{x}^{p} / L_{y} \rightarrow \infty$ or 0 . If $p$ is not known, it is not possible to proceed. In another context, a cellular automaton model with orthogonal biases has been formulated to mimic cross traffic flow 9]. A similar jamming transition has also been found. Whether the transition survives in the thermodynamic limit is likewise not investigated.

Motivated by these open questions, we study in this letter a three-state model with two inter-penetrating ASEP orthogonal to each other. By biasing the first (second) species along $+y(+x)$ direction, isotropic finite-size effects are ensured (i.e., effectively $p=1$ above) so that no ambiguity is associated with the thermodynamic limit, whereas the essential simplicity of previous models is maintained. Specifically, we consider a model on a two-dimensional (2D) square lattice of square geometry $L \times L$, with periodic boundary conditions. Occupation numbers $\left\{n_{x, y}\right\}$ specify the configuration, where $n_{x, y} \in\{0,1,2\}$ represents a vacancy, a type- 1 or a type- 2 particle at site $(x, y)$, respectively. Except hard-core exclusion, the particles are non-interacting. In simulations, a site and one of its nearest neighbors are randomly picked in turn. If and only if one of them is vacant, a jump occurs with rate: $p$ for a type- 1 particle hopping along $+y$, or a type- 2 particle hopping along $+x ; q$ for type- 1 along $-y$, or type- 2 along $-x$; and $r$ for type- 1 along $\pm x$, or type- 2 along $\pm y$. $L^{2}$ such trials constitute one unit of time (one sweep). Note that choosing $p>q \geq 0$ introduces the asymmetry, whereas $r>0$ provides transverse diffusions necessary for ergodicity (contrary to the traffic flow automaton[9] which has $r=0$ ). Using Metropolis rules and a parametrization by an "electric field" $\mathcal{E}[2]$, the rates become $p=1 / 4=r, q=e^{-\mathcal{E}} / 4$.

Simulation results: Simulations are done on wide ranges of parameters: $32 \leq L \leq$ $256,0.2 \leq \mathcal{E} \leq \infty$, with equal density of particles, $\bar{\rho}_{1}=\bar{\rho}_{2} \equiv \bar{\rho}$. While transitions between a uniform (U) phase and a strip (S) phase are expected as $\bar{\rho}$ is varied, a novel droplet phase (D) which is not present in previous models [7, 9] apppears in between 
(see Fig. 1). The three phases are characterized by different symmetries, with the localized droplet breaking translational invariance along both spatial directions and drifting steadily forward along its symmetry axis. That it is not an unstable or metastable state is revealed by the behavior of an order parameter, $\phi$ (essentially the Fourier amplitude of the density profile at a smallest wavevector[8]), which varies from one (compact strip) to zero (perfectly uniform). Fig. 1(a) depicts $\phi$ versus time $(t)$ in the steady state for $L=48, \mathcal{E}=\infty$, showing frequent jumps among the three phases. In fact, a histogram analysis shows that with those parameters the system is at a three-phase coexistence, i.e., at a triple point. Deviating from this point, we find: (a) At slightly different density, the $\mathrm{U}$ or $\mathrm{S}$ phase becomes dominant. (b) For smaller bias $\mathcal{E}$ with fixed $L, \phi(t)$ plots and histograms show that the triple point splits into two transitions, e.g., $\mathrm{S} \rightarrow \mathrm{D}$ at $\rho_{S D}$, then $\mathrm{D} \rightarrow \mathrm{U}$ at $\rho_{D U}$, as $\bar{\rho}$ decreases, with gradually widening gap $\left(\rho_{S D}-\rho_{D U}=0-0.02\right.$ as $\mathcal{E}=\infty-0.5$, for $L=48$ ). Diminishing first order characteristics — hysterisis and finite jumps in $\phi$ and currents - suggest that the transitions eventually turn continuous, consistent with the observation that $\rho_{S D}$ grows with decreasing $\mathcal{E}$, making the droplet longer and fuzzier until no longer distinquishable from a strip. (c) For larger $L$ at fixed $\mathcal{E}$, hysterisis becomes more pronounced while the transition points shift systematically downward.

These results remind us of a pure substance which has a triple point and a first order phase boundary ending at a critical point 10. Another possibility is to have a first and a continuous order phase boundary joining at a tri-critical point. Despite the obvious interest, however, we have not been able to map out the phase diagram completely. One difficulty is due to the above-mentioned decreasing transition densities for increasing system sizes, casting doubts on the survival of the transitions as $L \rightarrow \infty$. At the same time, growing hysterisis makes it harder to locate the transitions precisely. To understand these crucial finite-size effects, we now focus our attention on a continuum theory.

Mean-field theory: We begin by defining $P_{n n^{\prime} n^{\prime \prime} \ldots}^{y}(x, y, t)$ as an equal-time, joint probability at time $t$ for the locate state at sites $(x, y),(x, y+1),(x, y+2), \cdots$ to be $n, n^{\prime}, n^{\prime \prime}, \cdots$ where $n=0,1$ or 2 as above $\left(P_{n n^{\prime} n^{\prime \prime} \ldots}^{x}\right.$ likewise). Since particles only hop to nearest neighbors, it is easy to see, for $P_{n}(x, y, t+1)-P_{n}(x, y, t) \equiv \dot{P}_{n}$, that

$$
\begin{aligned}
\frac{1}{2} \dot{P}_{1}= & p\left[P_{10}^{y}(x, y-1)-P_{10}^{y}(x, y)\right]+q\left[P_{01}^{y}(x, y)-P_{01}^{y}(x, y-1)\right] \\
& +r\left[P_{10}^{x}(x-1, y)-P_{10}^{x}(x, y)+P_{01}^{x}(x, y)-P_{01}^{x}(x-1, y)\right] .
\end{aligned}
$$

The $1 / 2$ ensures the same time scale as in simulations, and lattice constant $\equiv 1$. $\dot{P}_{2}$ obeys a similar equation. An attempt to close this set of equations generates a hierarchy in the usual way. To proceed, we instead adopt a mean-field approximation in the following sense:

$$
P_{n n^{\prime}}^{y}(x, y) \approx P_{n}(x, y) P_{n^{\prime}}(x, y+1)\left[1+\Delta_{n n^{\prime}}^{y}(x, y)\right]
$$

with the asymmetric local correlations induced by biases accounted for by $\Delta_{n n^{\prime}}$ 's (similarly for $P_{n n^{\prime}}^{x}$ ). Clearly $\Delta_{12}^{y}>0, \Delta_{21}^{y}<0$ etc. Only those with $n \neq n^{\prime}$ are kept, as $\Delta_{n n} \ll 1$ is expected and well confirmed by simulation.

By taking the (naive) continuum limit of Eq. (11) via Taylor expansions, assuming smooth functions, we obtain from Eq. (1) the continuity equation $\dot{\rho}_{n}=-\nabla \cdot \mathbf{J}_{n}$ for 
density $\rho_{n}$, with the current

$$
\begin{aligned}
\mathbf{J}_{1}= & \hat{x} E_{1 x} \rho_{1} \rho_{2}+\hat{y}\left(E \rho_{0} \rho_{1}-E_{1 y} \rho_{1} \rho_{2}\right) \\
& -\hat{x} D_{\perp}\left[\left(1-\rho_{2}\right) \frac{\partial \rho_{1}}{\partial x}+\rho_{1} \frac{\partial \rho_{2}}{\partial x}\right]-\hat{y} D_{\|}\left[\left(1-\rho_{2}\right) \frac{\partial \rho_{1}}{\partial y}+\rho_{1} \frac{\partial \rho_{2}}{\partial y}\right],
\end{aligned}
$$

where the parameters are completely specified by the microscopics: $E=2(p-q)$, $E_{1 x}=2 r\left(\Delta_{21}^{x}-\Delta_{12}^{x}\right), E_{1 y}=2\left(p \Delta_{12}^{y}-q \Delta_{21}^{y}\right), D_{\|}=p+q$, and $D_{\perp}=2 r . \mathbf{J}_{2}$ can be obtained from $\mathbf{J}_{1}$ by switching the labels $1 \leftrightarrow 2$, and $x \leftrightarrow y$. Before we continue, note that the terms proportional to $E$ 's and $D$ 's represent physically drifts and diffusions respectively. There is an induced drift normal to the bias in spite of symmetric transverse jump rates. Similar but much weaker corrections in the diffusive pieces due to $\Delta$ 's are dropped in (3). In the case of $p=q$, or $\rho_{1}=0$, or $\rho_{2}=0$, the stationary state is a uniform product measure and Eq. (2) becomes exact with $\Delta=0$. Consequently, it is necessary to have two species driven to produce all the nontrivial behavior. Contrary to similar equations derived from entropy consideration[]], the microscopic origin of each term is made explicit.

Recall that there are three different steady states in simulations. While the uniform solution is trivial to find, the droplet requires solving the full $2 \mathrm{D}$ problem. Since the droplet phase occupies an extremely narrow range in $\bar{\rho}$ (i.e., $\rho_{S D} \approx \rho_{D U}$ ), for practical purposes we may focus on the uniform and the strip phase. By setting $\mathbf{J}_{n}=$ const, it is straightforward to show that the strip profiles satisfy

$$
\begin{aligned}
\frac{1}{\varepsilon_{0}} \rho_{1}^{\prime} & =\rho_{1}\left(1-\rho_{1}+\rho_{2}\right)-\frac{1}{\rho_{0}}\left(\varepsilon_{1} \rho_{1} \rho_{2}+C\right) \\
-\frac{1}{\varepsilon_{0}} \rho_{2}^{\prime} & =\rho_{2}\left(1+\rho_{1}-\rho_{2}\right)-\frac{1}{\rho_{0}}\left(\varepsilon_{1} \rho_{1} \rho_{2}+C\right)
\end{aligned}
$$

for equal $\bar{\rho}_{1}=\bar{\rho}_{2}=\bar{\rho}$, where $\varepsilon_{0}=\sqrt{2} E /\left(D_{\|}+D_{\perp}\right)=2 \sqrt{2}(p-q) /(2 r+p+q)$, $\varepsilon_{1}=\left(E_{1 x}+E_{1 y}\right) / E=\left[p \Delta_{12}^{y}+r \Delta_{21}^{x}-q \Delta_{21}^{y}-r \Delta_{12}^{x}\right] /(p-q), \rho_{n}^{\prime} \equiv d \rho_{n} / d u$, with the $u$ axis perpendicular to the strip, at a polar angle of $135^{\circ} . C=\sqrt{2} \mathcal{J} / E$ is a reduced current along $+u$, where $\mathcal{J}=\mathbf{J}_{1} \cdot(\hat{y}-\hat{x}) / \sqrt{2}$. $u$ and $\mathcal{J}$ have the same units as in simulations.

Using the data for $\Delta$ 's, we find a remarkable result: $\varepsilon_{1} \approx 1-2 \bar{\rho}=\bar{\rho}_{0}$ for arbitrary $\mathcal{E}, L$ and $\bar{\rho}$ in the uniform phase (i.e., all data fall on one universal line). While apparently $\Delta \rightarrow 0$ (so does $\varepsilon_{1}$ ) as $\bar{\rho}_{0} \rightarrow 0$, we have yet an explanation for the full, universal behavior. In line with a mean-field approach, we assume it remains to hold locally and replace $\varepsilon_{1}$ by $\rho_{0}(u)$ in Eqs. (4) and (5). Agreement between the predictions and simulations provides a posteriori justification [11. Then Eqs. (44) and (5) contain no adjustable parameter and imply the scaling form

$$
\rho_{n}(u, \mathcal{J}, L, \mathcal{E})=f_{n}\left(u \varepsilon_{0}, \mathcal{J} / E, L \varepsilon_{0}\right) .
$$

Unlikely to be obtained in closed forms, the functions $f_{n}$ 's are determined by numerically solving the coupled Eqs. (44) and (5). One stringent test of the theory is to compare these functions with those in simulations. Fig. 2 shows a typical test of not only $f_{n}$ but also the scaling in $L$. With no adjustable parameter, the agreements are impressive verifications of our approach. Deviation becomes noticeable only in the small $L \varepsilon_{0}$ regime. Furthermore, by (6),$(\sqrt{2} / L) \int d u \rho_{n}(u)=\bar{\rho}\left(\mathcal{J} / E, L \varepsilon_{0}\right)$, or equivalently

$$
C=\sqrt{2} \mathcal{J} / E=g\left(\bar{\rho}, L \varepsilon_{0}\right)
$$


This implies the entire current-density relation (along with the transitions) is the same for different $L$ and microscopic $\mathcal{E}$, provided $L \varepsilon_{0}$ is fixed. Fig. 3 confirms this prediction. The $\bar{\rho}$ dependence of $g$ also compares favorably with simulations, except at small $L \varepsilon_{0}$. This is consistent with the observed transitions gradually turning continuous at small biases, as fluctuations ignored in the equations become significant.

Eq. (7) allows an investigation of the thermodynamic limit. The transition point $\bar{\rho}_{c}(L, \mathcal{E})$ is taken to be the minimum $\bar{\rho}$ allowed for the strip solution of a given $L \varepsilon_{0}$ (i.e., at $d C / d \bar{\rho}=\infty$; cf. Fig. 3). It is a function of $L \varepsilon_{0}$ alone. $\lim _{L \varepsilon_{0} \rightarrow \infty} \bar{\rho}_{c}\left(L \varepsilon_{0}\right)$ then decides whether the transition survives or not. Fig. 4 shows, for both simulations 12 and theory, that $\bar{\rho}_{c} \sim\left(L \varepsilon_{0}\right)^{-0.82(1)}$, and strongly suggests $\bar{\rho}_{c} \rightarrow 0$ as $L \varepsilon_{0} \rightarrow \infty$.

These results have far reaching implications. If $\lim _{L \varepsilon_{0} \rightarrow \infty} \bar{\rho}_{c}=0$ indeed holds, then finite-density transition will occur generically only for infinitesimal bias (i.e., $\mathcal{E} \propto 1 / L)$. It is hasty, however, to conclude that the transition would actually be extinct for finite $\mathcal{E}$. Seldom found in equilibrium, this situation is by no means unfamiliar in non-equilibrium steady states. A case of intense interest is the well studied 1D limited local sandpile models of self-organized criticality [13]. Criticality of such models is controlled by the trough which has density $\rho_{t}$ vanishing as $L^{-1 / 3}$ [14, but the number of troughs $L \rho_{t} \rightarrow \infty$ as $L \rightarrow \infty$. Analogously, our model has the strip width $\xi \sim L \bar{\rho}_{c} \rightarrow \infty$ but $\xi / L \rightarrow 0$ (hence $\xi$ is a mesoscopic length) as $L \rightarrow \infty$, so that, like the sandpiles, the transition remains well-defined for all $L[15]$. We suspect other models have similar features, but the corresponding analysis is hindered by anisotropies[7] or by the lack of ergodicity[9].

To conclude, we have studied systems governed by asymmetric diffusion and twospecies exclusion as simple models exhibiting non-equilibrium phase transitions. Our primary results are: (a) There exists multiple phases in spite of their apprarent simplicity; (b) a quantitatively successful theory with known microscopic dependence has been developed, which could be improved to handle fluctuations by adding noise terms, and extended to more complicated models or related problems, such as with inter-particle interactions; and (c) the survival of the associated transitions in the thermodynamic limit is addressed for the first time, and found to be parallel to certain class of model sandpiles. Finally, open questions such as the mechanism of stability for the droplet, details of the phase diagram and issues of critical exponents at small biases are of significant interests to the general study of non-equilibrium phase transitions.

Acknowledgments: The author thanks Prof. J.L. Lebowitz for an illuminating discussion, and Dr. C.K. Chan for comments on the manuscript. Support from the National Science Council of ROC under grant number NSC83-0208-M-001-052 is gratefully acknowledged.

Note added: At the completion of the manuscript, we received a preprint from Vilfan et al. [16], who obtained mean-field exact solutions for the strip phase in the oppositely biased two-species model[[]. Their results agree qualitatively with ours, including a tentative tri-critical point, but the large- $L$ limits have not been explored. 


\section{References}

[1] See, e.g., H. Spohn, Large-Scale Dynamics of Interacting Particles, Texts and Monographs Phys. (Springer-Verlag, Berlin, 1991)

[2] See, e.g., B. Schmittmann and R.K.P. Zia, in Phase Transitions and Critical Phenomena, edited by C. Domb and J. L. Lebowitz (Academic, New York), to appear

[3] D.A. Huse, C.L. Henley, and D.S. Fisher, Phys. Rev. Lett. 54, 2924 (1985); M. Kardar, G. Parisi, and Y.C. Zhang, ibid 56, 889 (1986)

[4] J. Krug and L.-H. Tang (unpublished)

[5] J. Krug, Phys. Rev. Lett. 67, 1882 (1991); B. Derrida, E. Domany, and D. Mukamel, J. Stat. Phys. 69, 667 (1992)

[6] B. Derrida, S.A. Janowsky, J.L. Lebowitz, and E.R. Speer, Europhys. Lett. 22, $651(1993)$

[7] B. Schmittmann, K. Hwang and R.K.P. Zia, Europhys. Lett. 19, 19 (1992); K. Bassler, B. Schmittmann, and R.K.P. Zia, ibid 24, 115 (1993)

[8] K.-t. Leung, Phys. Rev. Lett. 66, 453 (1991) and Int'l J. of Mod. Phys. C3, $367(1992)$

[9] O. Biham, A. Middleton, and D. Levine, Phys. Rev. A 46, R6124 (1992)

[10] See, e.g., L.E. Reichl, A Modern Course in Statistical Physics, (Univ. of Texas, Austin, 1980)

[11] Setting $\varepsilon_{1}=$ const produces the same qualitative features but with less quantitative successes.

[12] For $L \varepsilon_{0}>45, \rho_{S D}, \rho_{D U}$ and their counterparts for reverse transitions begin to deviate. Since our predictions come from the strip solution, $\rho_{S D}\left(\approx \rho_{D U}\right.$ for all $\left.L \varepsilon_{0}\right)$ is used to compare with theory.

[13] L. P. Kadanoff, S. R. Nagel, L. Wu and S.-m. Zhou, Phys. Rev. A 39, 6524 (1989); P. Bak, C. Tang, and K. Wiesenfeld, Phys. Rev. Lett. 59, 381 (1987)

[14] J. M. Carlson, J. T. Chayes, E. R. Grannan, and G. H. Swindle, Phys. Rev. A 42, $2467(1990)$

[15] K.-t. Leung, Phys. Rev. E 48, R2331 (1993)

[16] I. Vilfan, R.K.P. Zia, and B. Schmittmann, (unpublished) 


\section{Figure Captions}

1. (a) Order parameter at three-phase coexistence for $L=48, \mathcal{E}=\infty$, $\bar{\rho}=0.1623$ : large, intermediate and small $\phi$ value corresponds to strip, droplet and uniform phase, respectively; (b) a typical strip for $L=128$, $\bar{\rho}=0.076$; and (c) a typical droplet for $L=128, \bar{\rho}=0.074$. Light (dark) particles drift to the right (top).

2. Finite-size scaling of density profiles at fixed $L \varepsilon_{0}=23.65$ (or fixed $C$ ) and $\bar{\rho}=0.36: L=32(\square), 48(\triangle), 64(\circ), 128(\times), 256$ (dashed line), and theory (solid line) [cf. Eq. (6) ] with no free parameter.

3. Scaling of current [Eq. (7)]. Thin line is $\bar{\rho}(1-\bar{\rho})(1-2 \bar{\rho})$ for uniform phase [from (身]. Branches are for strip phase at various $L \varepsilon_{0}$ : (a) 6.445, $L=48(\circ)$; (b) 14.81, $L=32(\square), 48(\triangle), 64(\nabla)$; (c) 23.65, $L=48(\bullet)$, 64( $\triangle)$, 128(口), 256(×); (d) 33.84, $L=48(\nabla)$; (e) 45.26, $L=48(\square)$; (f) $75.43, L=80(\nabla)$. Note confirmed scaling in (b) and (c). Heavy lines are theoretical predictions for (b) to (f).

4. Large- $L$ behavior of transition density: theory[cf. Fig. 3]( $\triangle)$; $\operatorname{data}(\bullet$ with error bars); linear fit(dotted line). 


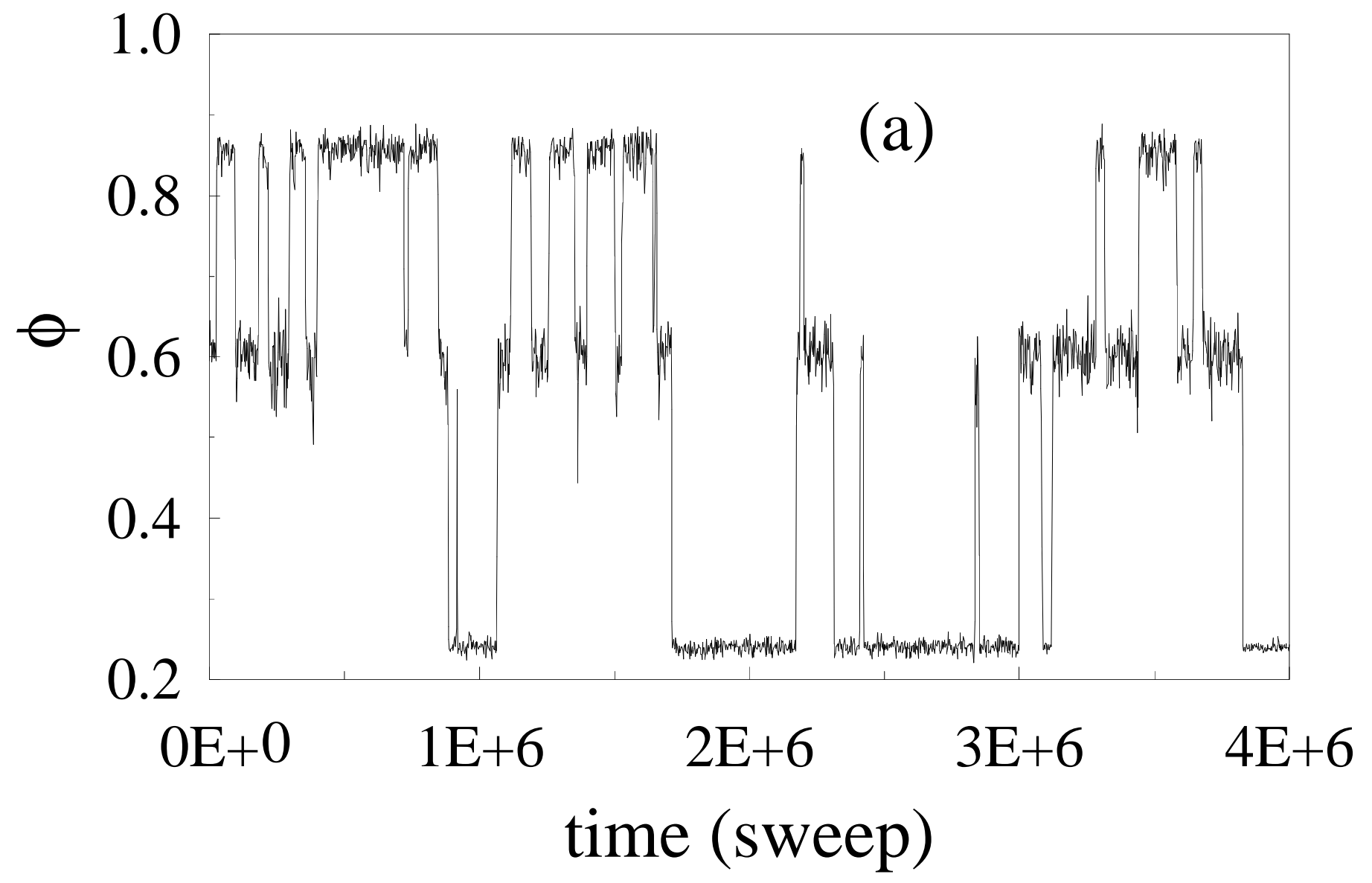




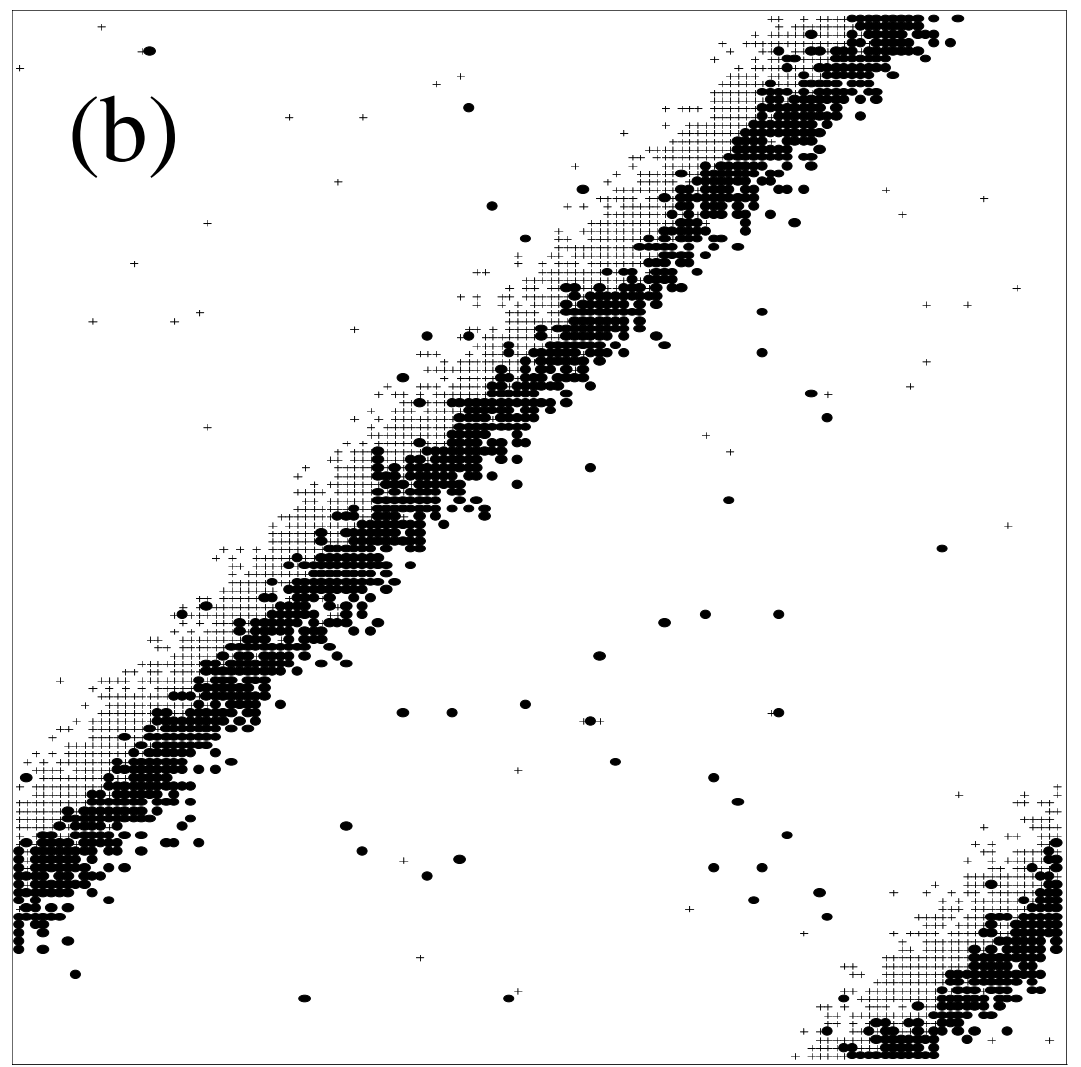




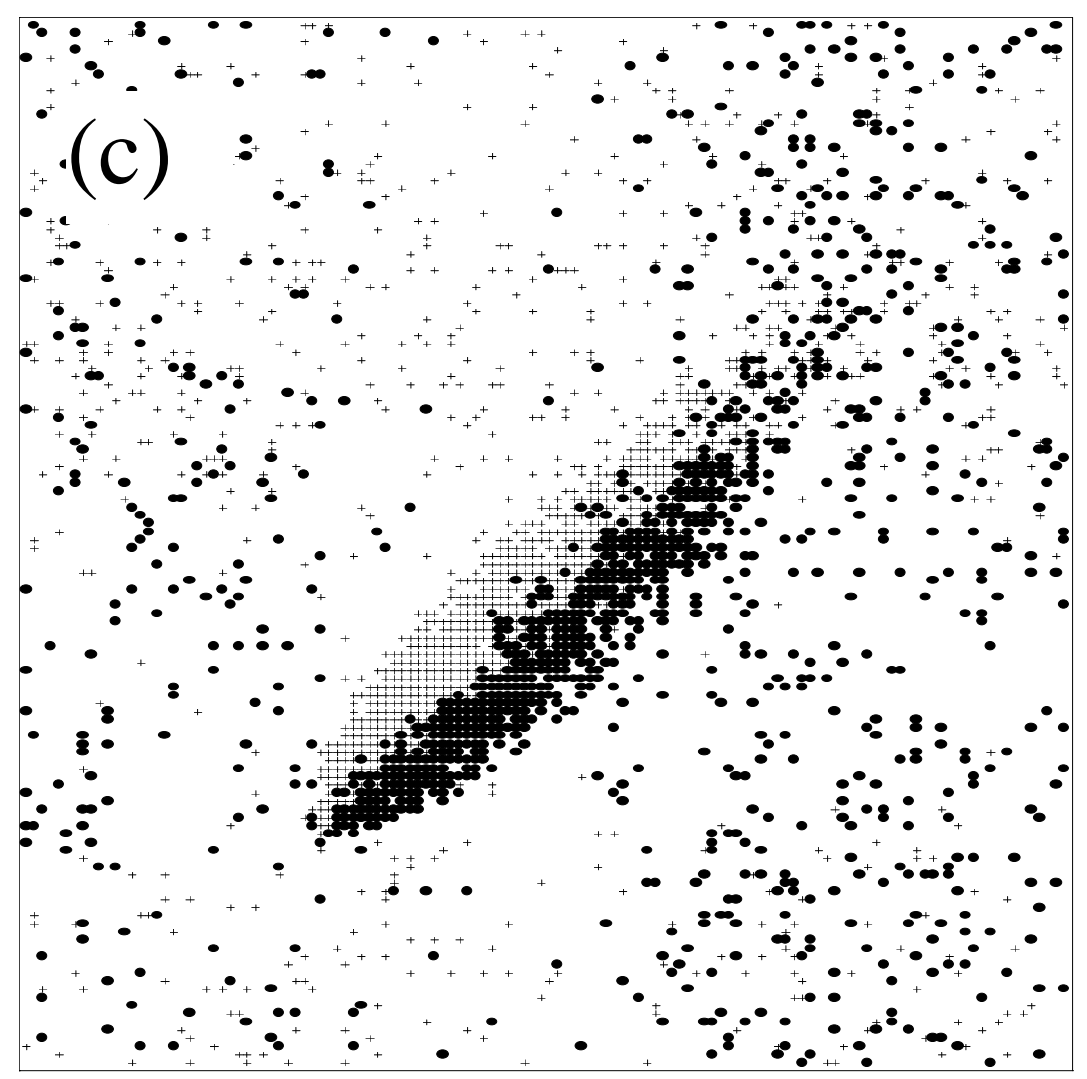




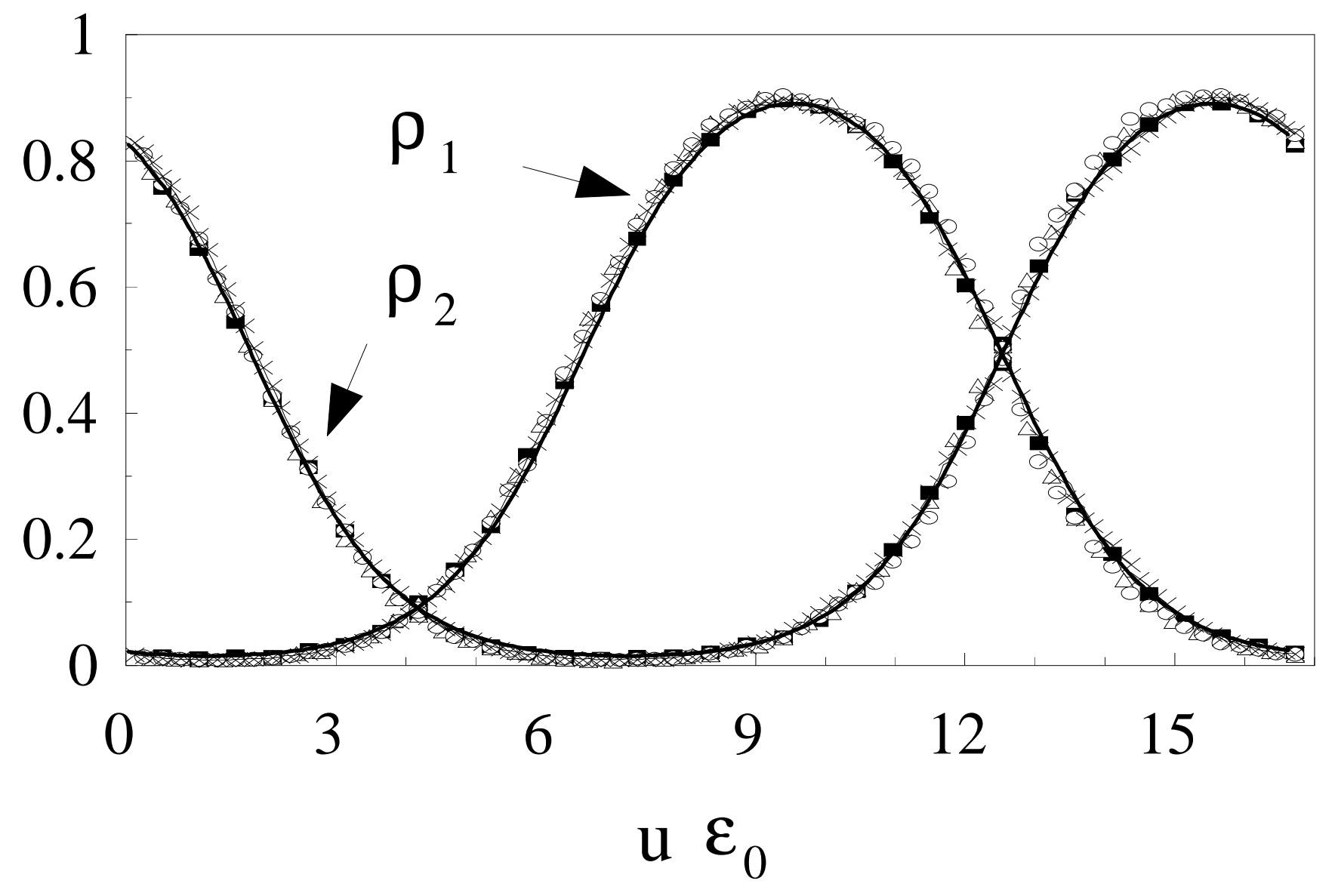




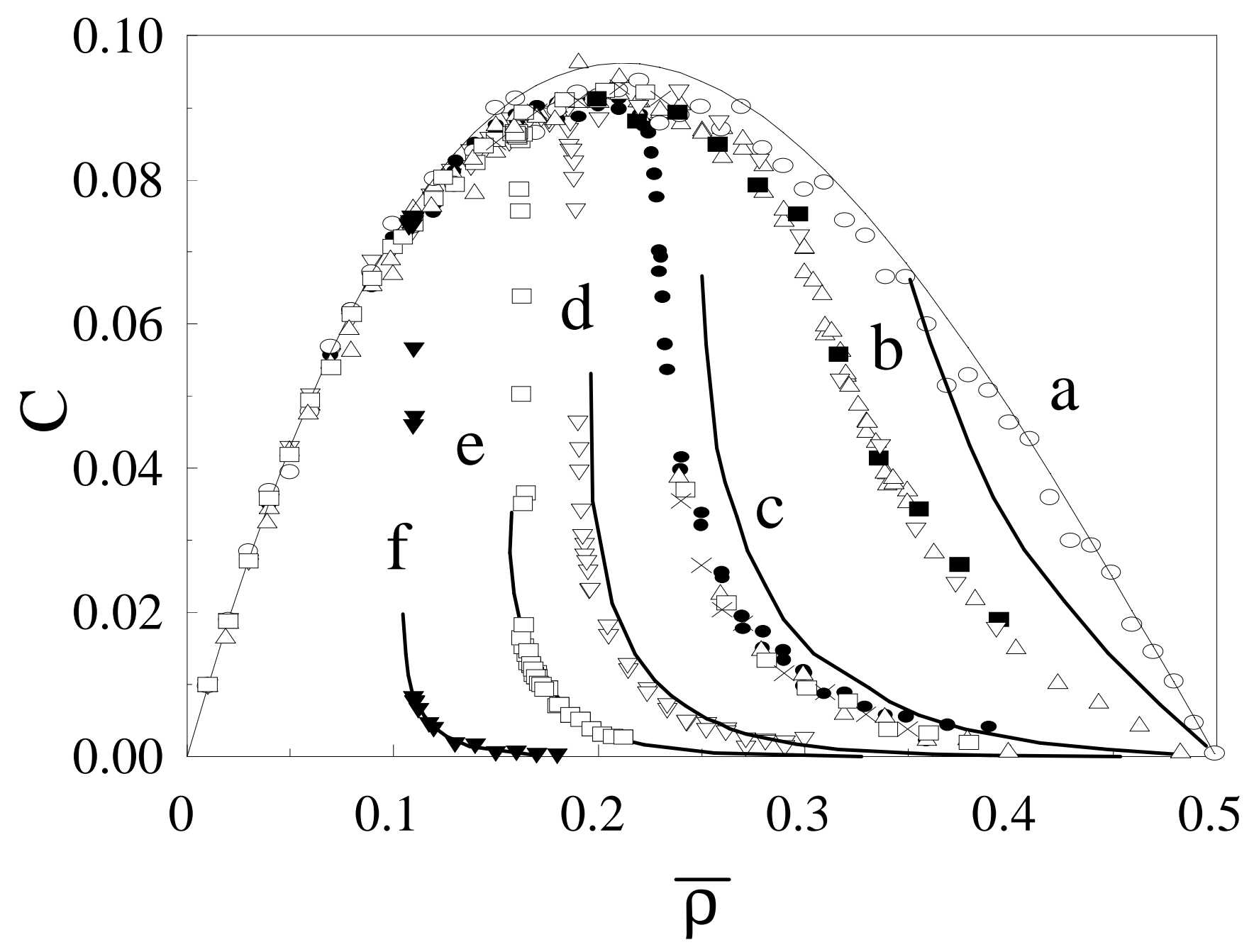




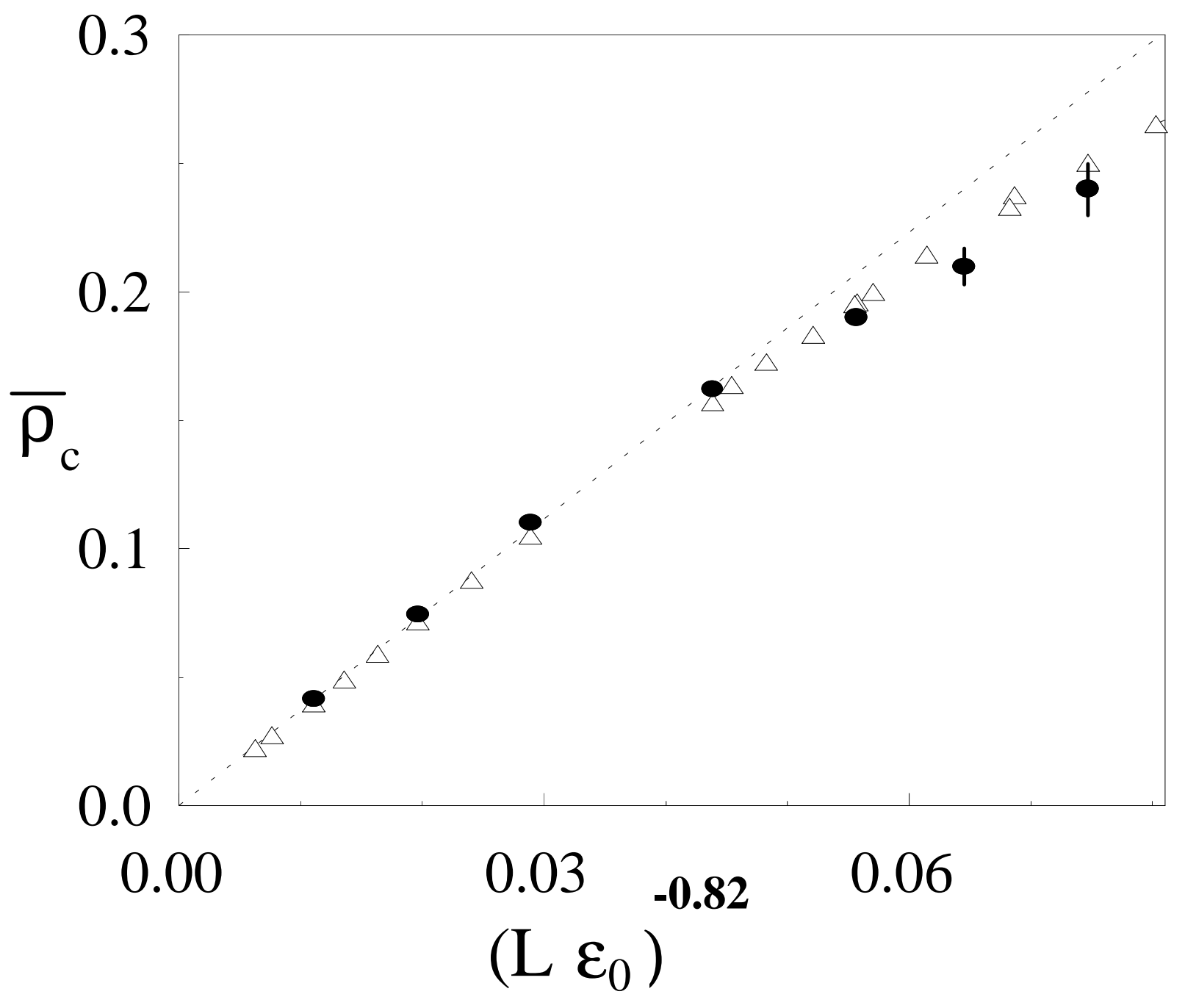

